\title{
Stem Cells: Umbilical Cord/Wharton's Jelly Derived
}

\author{
John T. Walker, Armand Keating, and John E. Davies
}

\section{Contents}

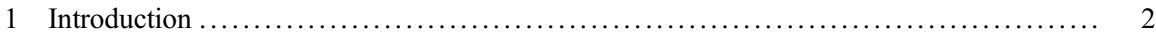

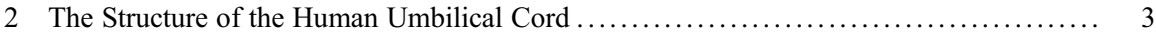

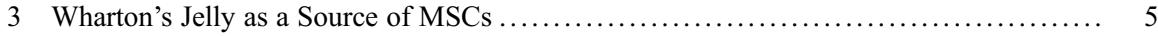

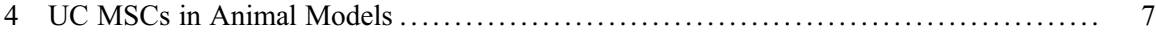

4.1 Inflammatory Diseases ................................................ 9

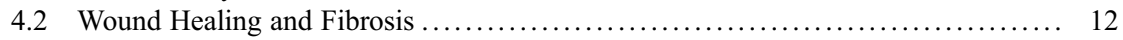

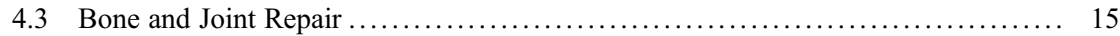

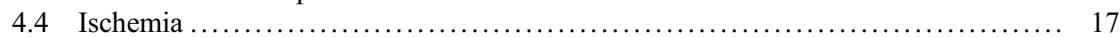

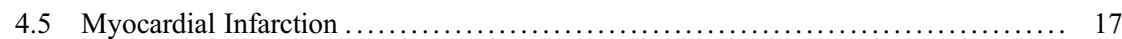

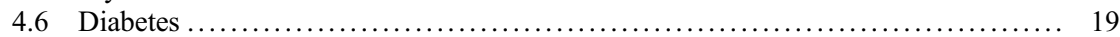

\section{J. T. Walker}

Anatomy \& Cell Biology, Schulich School of Medicine and Dentistry, The University of Western Ontario, London, ON, Canada

e-mail: jwalk29@uwo.ca
A. Keating
Institute of Biomaterials and Biomedical Engineering, Toronto, ON, Canada
University of Toronto, Toronto, ON, Canada
Cell Therapy Program, University Health Network, Toronto, Canada
Arthritis Program, Krembil Research Institute, University Health Network, Toronto, Canada
Princess Margaret Cancer Centre, University Health Network, Toronto, ON, Canada e-mail: armand.keating@uhn.ca

J. E. Davies ( $ه)$

Institute of Biomaterials and Biomedical Engineering, Toronto, ON, Canada

Faculty of Dentistry, Toronto, ON, Canada

e-mail: jed.davies@utoronto.ca 


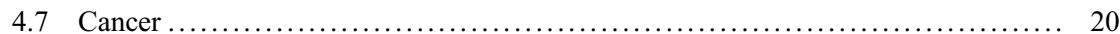

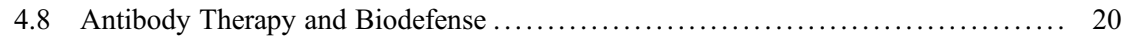

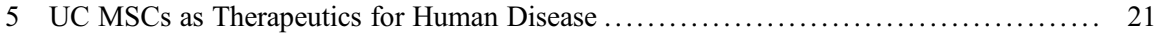

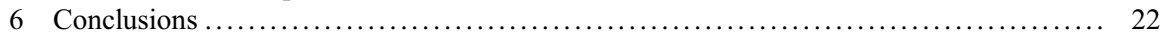

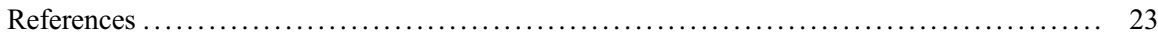

\begin{abstract}
As a commonly discarded tissue, the umbilical cord contains a rich source of mesenchymal stromal cells, which are therefore obtained non-invasively. As a perinatal population, replicative senescence is delayed and cell expansion is expedited, enabling collection of many clinically relevant doses from a single donor cord at low passage numbers. In this chapter, we will discuss the structure of the umbilical cord and the various stromal populations contained within that have been described. We also highlight the lack of consensus on both anatomical descriptors of the cord tissue, and standardized isolation techniques for these different populations, which together with insufficient methodological transparency may be hampering progress within the field. We then review the basic and preclinical models of disease that have been targets of umbilical cord-derived mesenchymal stromal cells. Finally, we close with a discussion of their use in clinical trials.
\end{abstract}

\title{
1 Introduction
}

The human umbilical cord is increasingly being employed as a tissue source of cells for cell therapy. While cord blood has been used therapeutically since 1988, the harvesting of cells from the structural tissue of the cord dates from the first isolation of human umbilical vein endothelial cells in 1963 (Maruyama 1963), although in all studies they have been limited to laboratory experiments, or clinically related assays, rather than therapeutic uses. More recently, since 2009, cell populations harvested from the nonvascular tissues of the umbilical cord have been employed for many different clinical targets. While the exact cell populations isolated from the cord are often not evident, and potentially include multiple unique subpopulations as discussed below, they are all generally described as MSCs.

Most authors now define an MSC by the minimal criteria suggested by the International Society for Cellular Therapy (ISCT) elaborated in their position paper of 2006 (Dominici et al. 2006). In the latter, the term mesenchymal stromal cell (MSC), rather mesenchymal stem cell, was proposed since evidence of the selfrenewal and multi-lineage differentiation potential that define a stem cell were not generally provided by authors. We use the term MSC herein to describe the cell population derived from the connective tissue of the human umbilical cord, or Wharton's Jelly. But we would also point out that some authors have included the 
amniotic epithelium, the smooth muscle of the tunica media of the umbilical vessels, and even their endothelial linings in their harvested populations. Nevertheless, our focus herein will be on MSC populations harvested from the nonvascular tissue of the human umbilical cord, the basic and preclinical studies that have been carried out both in vitro but predominantly in vivo in animal models, and the range of clinical studies that have been initiated using these cells.

However, we start by briefly reviewing the structure of the human umbilical cord, and the context of this tissue source in light of all MSC tissue sources being employed in clinical studies.

\section{The Structure of the Human Umbilical Cord}

At term, the human umbilical cord is approximately $60 \mathrm{cms}$ long with an average diameter of $1.5 \mathrm{~cm}$. It has an outer covering of a single layer of amniotic epithelium and contains three vessels, a vein and 2 arteries, that are surrounded by a mucoid connective tissue called Wharton's Jelly (Wharton 1656). A single cross-section such as that in Fig. 1 illustrates the arrangement of these component parts. Importantly, in the human umbilical cord the vessels comprise only a tunica media and an endothelial lining. The role of the adventitia is borne by the Wharton's Jelly surrounding the vessels and known as the perivascular Wharton's Jelly. Distal to the perivascular jelly both cells and matrix become sparse, and clefts which contain only ground substance, are evident until the narrow cleft-free sub-amniotic zone immediately below the amniotic epithelium, which is commonly only one or two cells thick. We have recently described, elsewhere, the detailed anatomical structure

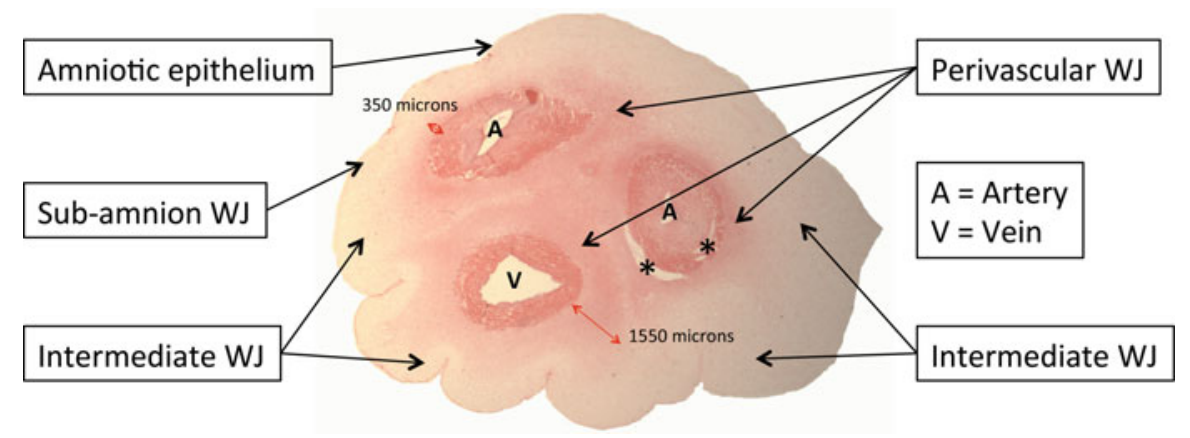

Fig. 1 A paraffin embedded cross-section of the human umbilical cord stained with hematoxylin and eosin. Shows an outer amniotic epithelium and three vessels contained within Wharton's Jelly (WJ), which extend from the tunica media of the vessels to the amniotic epithelium. Wharton's Jelly is denser in the perivascular zones, due to an increase in both cells and matrix, and measures 350-1550 microns deep in this sample (as marked). The paucity of staining in areas beyond the perivascular zones is, in part, due to the presence of clefts (visible when the image is enlarged) in the Intermediate WJ. The perivascular regions are separated by narrow regions of intervascular jelly that also contains clefts. The asterisks mark artifacts of preparation. Total Width $=15 \mathrm{~mm}$ 
of the human umbilical cord, its embryological derivation, together with some comparative anatomy for other commonly employed species (Davies et al. 2017). However, it is important to emphasize that until there is common agreement on terminology used to describe either the anatomy of the cord or the cell populations harvested, it will be difficult to make detailed comparisons between the increasing numbers of studies employing this important tissue source.

Can and Karahuseyinoglu identified six zones within the human umbilical cord: (1) the surface (amniotic) epithelium, (2) subamniotic stroma, (3) clefts, (4) intervascular stroma, (5) perivascular stroma, and (6) the vessels (Can and Karahuseyinoglu 2007). They considered only zone 4 to be Wharton's Jelly, although most authors would describe Wharton's Jelly to comprise all the tissue outside the tunica media of the three vessels and bounded by the amniotic epithelium (zones 2-5 inclusive). Schugar et al., who described the perivascular zone as having an average depth of 430 microns, showed that $45 \%$ of the cells in Wharton's Jelly are found in zone 5 (Schugar et al. 2009). Of these anatomical structures of the cord, the descriptions of zones 1, 3, and 6 are generally agreed upon by the majority of authors. However, the description of zone 5 (and thus also zone 4) is highly variable between authors and ranges from a layer only 2 cells thick (Kita et al. 2010) or less than 500 microns deep (Troyer and Weiss 2008; Coskun and Can 2015) to 750-2000 microns deep (Subramanian et al. 2015) or 350-1550 microns as shown in Fig. 1. Reference to Fig. 1 will also make it clear that the depth of zone 4 is approximately 350 microns and that the 6 zone classification takes no account of the regions labeled Intermediate WJ in Fig. 1, which are quite extensive, although not rich in cells. Table 1 accompanying Fig. 1 provides a summary of the anatomical structure and classification of the human umbilical cord. From a cell harvesting perspective, it is relatively easy to isolate the perivascular tissue for cell extraction by numerous methods. Also, with fine dissection, it is possible to separate the Sub-Amnion, about 150 microns of Wharton's Jelly, from both the underlying Intermediate WJ and Perivascular WJ, and the overlying Amniotic epithelium. However, it would be exceedingly difficult to isolate either the Intermediate WJ from both the SubAmnion WJ and the Perivascular WJ, or the Intervascular WJ (not marked in Fig. 1) from all other regions of the WJ.

These structural considerations are important since some authors have claimed to harvest cells from only selected zones of WJ, while others have reported that they extracted cells from Wharton's Jelly, without defining the exact tissue to which they are referring. We define Wharton's Jelly (see Table in Fig. 1) as all the mucoid tissue surrounding the vessels and extending to the underside of the enveloping amniotic epithelium. Furthermore, we define the perivascular tissue as that tissue, which is cell and matrix rich when compared to the remainder of Wharton's Jelly, and which surrounds the three vessels of the cord. This tissue has been previously referred to as the adventitia of the cord vessels (Nanaev et al. 1997). If the cord vessels are manually removed from the cord, after longitudinally opening or stripping the amniotic epithelium, the perivascular tissue remains adherent to the vessel wall as 
Table 1 The Anatomical Compartments of the Human Umbilical Cord. The Intervascular Wharton's Jelly (WJ) is not included here as an independent category, although it can be identified (see Fig. 1), because it cannot realistically be dissected from the Perivascular and Intermediate WJs, respectively. The endothelial cells of the tunica intima of the umbilical vein are known as human umbilical vein endothelial cells (HUVECs) while the Perivascular cells are known as human umbilical cord perivascular cells (HUCPVCs)

\begin{tabular}{l|l|l|l|l|l}
\hline The Human & Umbilical Cord & & Vessels & \\
\hline Amnion & Wharton's Jelly & $\begin{array}{l}\text { Perivascular } \\
\text { WJ } \\
\text { (intervascular } \\
\text { WJ) }\end{array}$ & Tunica Media & $\begin{array}{l}\text { Tunica } \\
\text { Intima } \\
\text { Epithelium } \\
\text { WJ }\end{array}$ & $\begin{array}{l}\text { Sub- } \\
\text { WJion }\end{array}$ \\
\hline $\begin{array}{l}1-3 \text { cells } \\
\text { thick }\end{array}$ & $\begin{array}{l}100-150 \\
\text { microns } \\
\text { thick }\end{array}$ & $\begin{array}{l}\text { Sparse matrix } \\
\text { and cells of } \\
\text { varying, } \\
\text { irregular, } \\
\text { dimensions }\end{array}$ & $\begin{array}{l}\text { 200-2000 } \\
\text { microns thick }\end{array}$ & $\begin{array}{l}\text { Two layers of } \\
\text { smooth muscle } \\
\text { orthogonally } \\
\text { arranged }\end{array}$ & $\begin{array}{l}\text { Single } \\
\text { layer of } \\
\text { endothelial } \\
\text { cells }\end{array}$ \\
\hline Cord "lining" & $\begin{array}{l}\text { The WJ } \\
\text { containing clefts }\end{array}$ & $\begin{array}{l}\text { A functional } \\
\text { adventia }\end{array}$ & & \\
\hline
\end{tabular}

we, and others (Farias et al. 2011), have shown. However, we also caution that the arrangement of the vessels, their girth, and that of the cord itself, and thus the dimensions of the various zones mentioned are variable, although with appropriate staining, easily visualized.

\section{Wharton's Jelly as a Source of MSCs}

The human umbilical cord is a rich source of MSCs. Indeed, it is the advent of MSC biology that has driven the increasing interest in umbilical cord tissue as witnessed by the increasing number of publications in the last two decades (see Fig. 2). This is in part due to the high cell yields, high colony forming unit frequencies, and short population doubling times that have been reported by many authors as illustrated in Fig. 3.

The yield of MSC from Wharton's Jelly has been shown to depend upon the method of cell extraction employed. Using a CFU-F assay as a surrogate MSC measure, this can be illustrated by a comparison of Sarugaser et al. (2005) and Lu et al. (2006). While the former reports a CFU-F of 1:300 at harvest from an isolated UC perivascular population (Sarugaser et al. 2009), Lu et al. reported a CFU-F of 1:1609 (Lu et al. 2006), from cell populations harvested from minced whole cord, which is still considerably higher than the values of 1:10,000 to less than 1:100,000 reported for bone marrow MSCs (Caplan 2007).

Thus, it is not surprising that Wharton's Jelly has become an important contributory source of cells for MSC clinical trials as seen in Fig. 4. Wharton's Jelly cells contribute to the almost $50 \%$ of MSC clinical trials that employ allogeneic cells. To our knowledge, there has been no report of autologous use of these cells to date. The first four trials were 


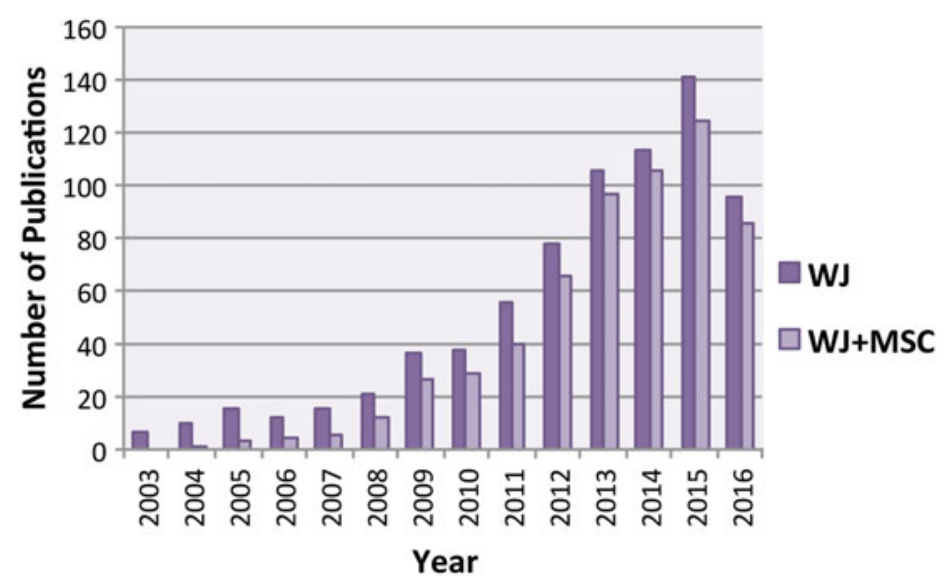

Fig. 2 A simple PubMed search using the term "Wharton's Jelly" returned seven publications in 2003 increasing to 141 in 2015. After 2007, the majority of papers also reference the term "MSC." (Note: 2016 for Jan-Oct only)

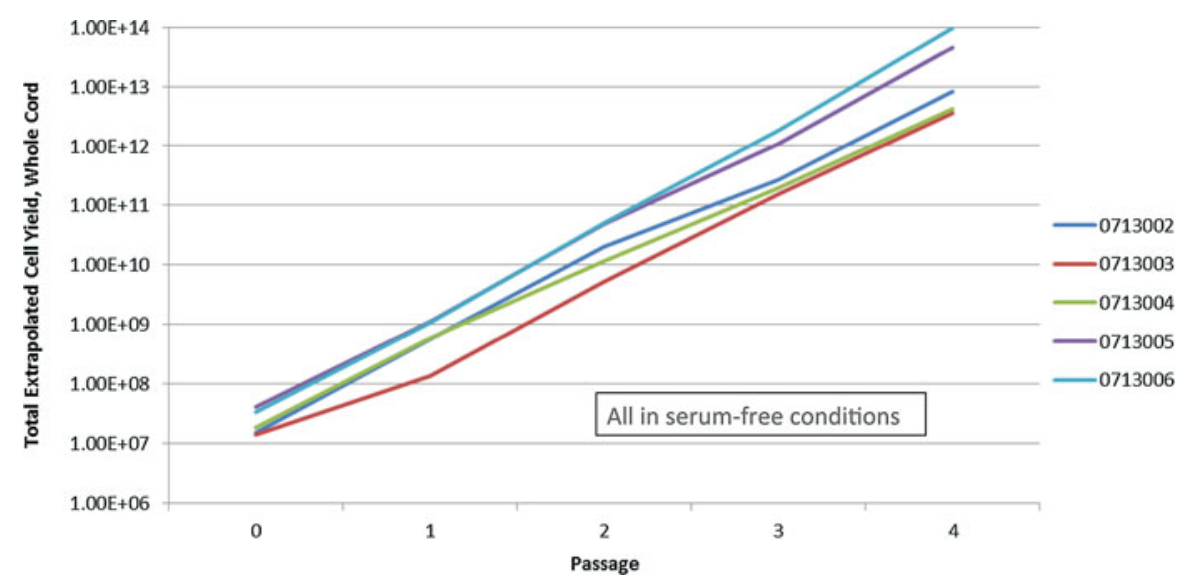

Fig. 3 Cell populations extracted from the perivascular tissue of the human umbilical cord can be rapidly expanded, at low passage, to vast numbers in serum-free conditions suitable for clinical use. (Data courtesy of Tissue Regeneration Therapeutics Inc.)

in 2009, but there are currently 68 individual trials (including those in more than one trial phase) registered on ClinicalTrials.gov. This, therefore, represents a significant proportion of the most recently registered allogeneic MSC trials.

These trials have been preceded by a large number of basic and preclinical studies. The following, while not comprehensive, provides examples of the breadth of these studies. Can and Karahuseyinoglu provided an excellent review in 2007 and several others have been published since. However, we append here an additional section on 

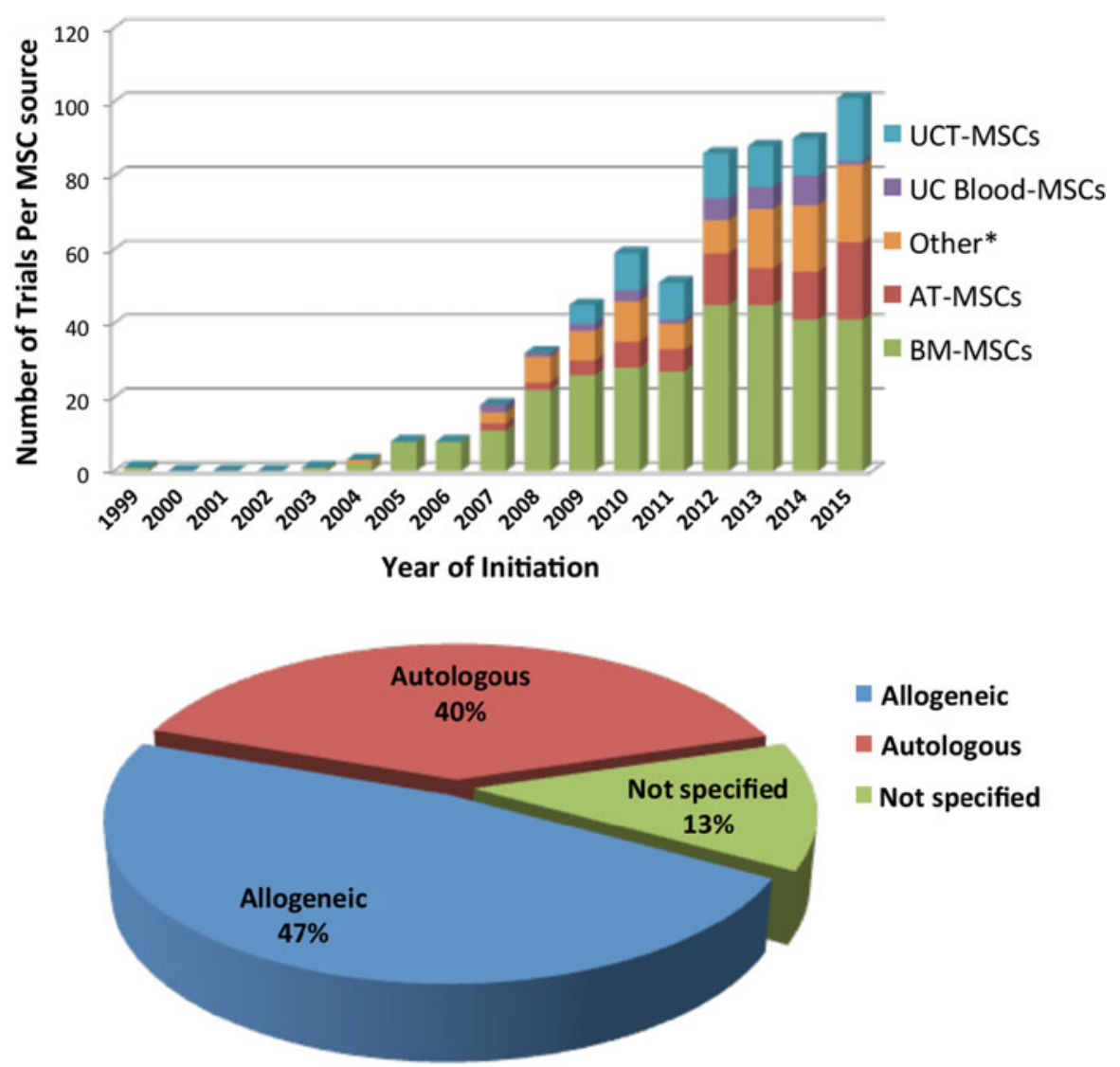

Fig. 4 Above: MSCs from multiple tissue sources have been employed in clinical trial to date. Below: Of these trials, $47 \%$ have used unmatched, allogeneic cells; $40 \%$ were autologous, while the remainder was not specified. (Data courtesy of Tissue Regeneration Therapeutics Inc. and obtained from ClinicalTrials.gov up to the end of 2014)

those papers that have been published as outcomes of the clinical trials mentioned above employing Wharton's Jelly cells.

\section{$4 \quad$ UC MSCs in Animal Models}

While there is still little known about the development and origins of MSCs from the umbilical cord, their utility in therapeutics has been reported for a diverse range of maladies in animal models. Of critical importance, like all described MSC populations, UC-MSCs display an immune-privileged phenotype. Their responsive and adaptable anti-inflammatory, anti-apoptotic, and pro-angiogenic nature are often 
cited as sources for their wide-ranging utility. Additionally, their rapid ex vivo expansion and capacity for a high number of population doublings allows for rapid harvest of clinically relevant doses as well as the ability to genetically modify these cells, turning them into biological factories which can be employed in situ. Here we will review several of the animal models in which umbilical cord MSCs have been studied.

Before reviewing these animal models, we consider it critical to emphasize the difficulty in making comparisons between studies when the methods of cell extraction are not provided in sufficient detail, or the zones of the cord from which cells are extracted are either not or differentially defined. To illustrate this, in their otherwise comprehensive paper, Subramanian et al. (2015) specifically chose to undertake a comparative characterization of cells derived from various compartments of the human umbilical cord. They compared cell populations from the amniotic membrane, the sub-amnion or "cord lining," the intervascular Wharton's Jelly, and the perivascular region. Histology was used to illustrate the various regions with the perivascular area labeled in similar manner to that in Fig. 1 herein. However, they provided no specific details of how they isolated cells from these different areas. Rather, they stated that the cells were isolated "using published established derivation protocols for each compartment" and list seven previously published papers for the methods. The latter are compared in Table 2. Within these references, one did not

Table 2 The papers referenced by Subramanian et al. (2015) to identify the method of isolation of cells from the various regions of the human umbilical cord

\begin{tabular}{l|l|l}
\hline $\begin{array}{l}\text { Ref\# from } \\
\text { Subramanian et al. }\end{array}$ & $\begin{array}{l}\text { Ref. in } \\
\text { Bibliography }\end{array}$ & Method \\
\hline 3 & $\begin{array}{l}\text { Sarugaser } \\
\text { et al. (2005) }\end{array}$ & $\begin{array}{l}\text { Cord: Amnion opened, each vessel with surrounding } \\
\text { WJ pulled away. Vessels tied, looped, WJ collagenase } \\
\text { digested from vessels }\end{array}$ \\
\hline 4 & $\begin{array}{l}\text { Ilancheran } \\
\text { et al. (2007) }\end{array}$ & $\begin{array}{l}\text { Cord not used. Amnion separated from Chorion to } \\
\text { isolate human amniotic epithelial cells (hAECs) }\end{array}$ \\
\hline 5 & $\begin{array}{l}\text { Troyer and } \\
\text { Weiss (2008) }\end{array}$ & $\begin{array}{l}\text { REVIEW: focuses on phenotype of UC-derived cells. } \\
\text { Provides no cell derivation protocols. Note: Cartoon of } \\
\text { perivascular region is limited to a thin surrounding of 1 } \\
\text { artery and vein - thus different to Subramanian et al. } \\
\text { (2015) }\end{array}$ \\
\hline 6 & $\begin{array}{l}\text { Schugar et al. } \\
(2009)\end{array}$ & $\begin{array}{l}\text { Cord: Whole pieces used either for (i) explant cultures } \\
\text { or (ii) for enzymatic digestion in either dispase or } \\
\text { collagenase }\end{array}$ \\
\hline 7 & $\begin{array}{l}\text { Kita et al. } \\
\text { (2010) }\end{array}$ & $\begin{array}{l}\text { Cord: Opened and WJ identified with phenol red, then } \\
\text { scraped off while outer envelop provided sub-amnion. } \\
\text { Note: perivascular region comprises a 2 cell thick layer }\end{array}$ \\
\hline 15 & $\begin{array}{l}\text { Fong et al. } \\
(2010)\end{array}$ & $\begin{array}{l}\text { Cord: Cut open and inner surface laid into collagenase/ } \\
\text { hyaluronidase solution without removing vessels. WJ } \\
\text { subsequently scraped into fresh medium }\end{array}$ \\
\hline 18 & $\begin{array}{l}\text { Bosch et al. } \\
(2012)\end{array}$ & $\begin{array}{l}\text { Cord: Cut into small pieces and seeded as either single } \\
\text { or multiple pieces for explant cultures }\end{array}$ \\
\hline
\end{tabular}


employ umbilical cord, while another was a review article that provided no specific cell derivation protocols; two papers diced whole cord tissue for explant cultures where every tissue in the cord would have been represented; two removed Wharton's Jelly by different means, neither of which distinguished between different regions of the cord and one specifically isolated cells from the perivascular jelly. It is thus impossible to determine how Subramanian et al. (ibid) identified the cells originating in the various regions of Wharton's Jelly. This lack of information is not uncommon in the studies quoted in the following sections.

\subsection{Inflammatory Diseases}

Several studies have shown that umbilical cord (UC)-MSCs share anti-inflammatory properties with their bone-marrow derived counterparts in vitro (Ennis et al. 2008; Payne et al. 2013; Donders et al. 2015). Consequently, UC-MSCs have been investigated in a range of diseases linked with excessive inflammation. Many of these diseases involve an improper balance between pro- and anti-inflammatory cells and their regulators. Ideally, a therapeutic could modify this imbalance to ameliorate tissue damage without significantly impairing the host response to invading pathogens. With the ability to respond and adapt to their environment, MSCs hold promise in these indications.

Autoreactivity of $\mathrm{B}$ and $\mathrm{T}$ cells with proteins of the myelin sheath has been suggested to be at the root of multiple sclerosis (MS) (McQualter and Bernard 2007; Steinman 2014). Experimental autoimmune encephalomyelitis (EAE) is an inducible animal model used to mimic this autoimmune reaction in MS. UC-MSCs have been explored in these models to limit the autoimmune destruction and improve regeneration of the myelin sheath. However, across three independent studies, the results have been variable. In a comparison of different MSC origins, Payne et al. showed that while bone marrow (BM)-MSCs display a greater anti-inflammatory response in vitro, only adipose derived (Ad)-MSCs and UC-MSCs significantly reduced the clinical score of mice compared to a PBS injected control (Payne et al. 2013). Still, there were no significant differences between any of the MSC groups, and improvements, which were only modest, could only be attained if cells were injected prior to the onset of symptoms (Payne et al. 2013). The authors linked the discrepancy between in vivo performance and in vitro anti-inflammatory response to differences in cell homing. Importantly, UC-MSCs and Ad-MSCs expressed a wider range of receptors involved in chemotaxis, as well as adhesion molecules used for extravasation at sites of injury when compared to BM-MSCs. Although UC-MSCs were not tested for homing capacity, they showed that Ad-MSCs could enter the CNS whereas BM-MSCs were unable to do so. Conversely, there were two studies showing minor improvements following UC-MSC administration, if injected following the onset of symptoms. Donders et al. found that the disease burden could be lessened, but only temporarily following UC-MSC injection whether injected at the onset of symptoms or even after a prolonged duration of symptoms (Donders et al. 2015). Notably, the improvements were not permanent and returned to levels of 
controls over time. In a study with the most promising results, Liu et al. showed lasting improvements in animals injected with UC-MSCs after the onset of disease (Liu et al. 2013). These results were further supported with improved histological outcomes and even improved remyelination. These differences between studies, which may in part be due to differences in the disease model (Donders et al. 2015), emphasize a need for further exploration into the use of UC-MSCs for EAE. Of interest, in another approach to improve EAE progression, Agah et al. (2013) investigated treatment with oligodendrocyte progenitor cells derived from UC-MSC precursors (Agah et al. 2013). When these differentiated cells were injected prior to the onset of symptoms, clinical grade of EAE was improved to the end of the study and the extent of demyelination was reduced.

Crohn's disease and ulcerative colitis, together making up the inflammatory bowel diseases (IBD), involve excessive immune responses to inappropriate targets within the digestive tract including the resident gut flora (Bouma and Strober 2003). Although the etiology of IBD is unknown, animal models have been developed to mimic the inflammation within the digestive tract and have provided much insight into these diseases. Once again, chosen for their global anti-inflammatory properties, MSCs are a prime candidate for treating IBD. UC-MSCs have proven to be useful in ameliorating chemical-induced colitis in mice (Liang et al. 2011; Lin et al. 2015). In two independent studies, UC-MSCs were shown to home to sites of inflammation within the colon, reduce the inflammatory response, and decrease symptoms of colitis (Liang et al. 2011; Lin et al. 2015). In both cases, this was confirmed through histological analysis. Interestingly, another group has sought to improve upon the natural benefits of UC-MSCs for this indication by enhancing their immunosuppressive effects. Pretreatment of UC-MSCs with IL-1 $\beta$ (Fan et al. 2012) or genetic modification with an IFN $\gamma$ expression construct (Chen et al. 2015) both improved treatment efficacy over unmodified UC-MSCs. Priming UC-MSCs with IL-1 $\beta$ led to improved homing as well as a modified immune response promoting a greater antiinflammatory response through M2 macrophage polarization and driving T cells to a Th2 phenotype (Fan et al. 2012). The IFN $\gamma$ expression construct was, similarly, shown to promote a more regenerative immune response and was also shown to reduce $\mathrm{T}$ cell activation in co-culture (Chen et al. 2015). Conversely, no changes to cell homing were reported in the IFN $\gamma$ group.

Ex vivo lung perfusion (EVLP) is a technique designed to increase the available pool of donor lungs for transplantation by providing a platform to test the functionality of typically discarded lungs (Machuca and Cypel 2014). Conventional transplant requires specific criteria for donor lungs while much of the available pool, not meeting these criteria, are discarded without any functional assessment. Moreover, EVLP can, in fact, improve lung condition during the assessment period. One previously unavailable benefit to this technique is that it allows for modification of the lungs during ex vivo perfusion. Accordingly, treatment with UC-MSCs has been explored as a mechanism to reduce inflammation from ischemia-reperfusion injury as well as decrease the host alloimmune response following transplantation (Mordant et al. 2016). From early ex vivo experiments measuring the effects of human UC-MSC perfusion into pig lungs damaged by prolonged cold ischemic 
storage, Mordant et al. found modest functional improvements in the form of enhanced static compliance of these damaged lungs. Moreover, a significant decrease of the pro-inflammatory cytokine, IL-8, within the lung perfusate was observed along with increased parenchymal VEGF. Although these are only early findings, they show a great deal of promise for enhancing the outcomes of EVLP reliant lung transplantations.

Sepsis can develop from a range of uncontrolled infections, which lead to specific antigens entering the systemic circulation, inducing an excessive and destructive immune response (Cohen 2002). If this is not controlled, it can result in organ failure. Furthermore, sepsis is the most common cause of acute respiratory distress syndrome (ARDS) which is associated with high patient mortality (Kim and Hong 2016). In both cases, systemic delivery of MSCs has been investigated to produce a long-term immunomodulatory environment within the host. Initially, BM-MSCs were compared to UC-MSCs in a cecal ligation and puncture model of sepsis (Chao et al. 2014). While treatment with UC-MSCs led to the greatest survival compared to BM-MSCs and PBS control, none of the differences were significant. Conversely, UC-MSCs, and to an equal extent, BM-MSCs, led to increased levels of circulating regulatory $\mathrm{T}$ cells, and higher regulatory $\mathrm{T}$ cell/T cell ratios. Additionally, circulating levels of IL-6 and TNF $\alpha$ were decreased following treatment with MSCs. In a second study using the same model, UC-MSCs were shown to only improve survival when co-administered with antibiotic, when compared to a PBS plus antibiotic treated control (Wu et al. 2016). Consistent with the previous study, $\mathrm{TNF} \alpha$ and IL-6 levels were significantly reduced in the UC-MSC administered animals compared to PBS controls, along with MCP-1 and IFN $\gamma$. Moreover, the anti-inflammatory cytokine IL-10 was upregulated in the UC-MSC group. These data display the strong systemic anti-inflammatory capabilities of UC-MSCs in these models. UC-MSCs have also shown efficacy in treating animal models of bacterial associated ARDS (Masterson et al. 2015). In a model of LPS-induced ARDS, Sun et al. showed an increase in inflammatory cytokines IFN $\gamma, \mathrm{TNF} \alpha$, and MIP-2 following intratracheal administration of LPS (Sun et al. 2011). Each of these were significantly reduced in both plasma and bronchoalveolar lavage (BAL) fluid in groups treated with UC-MSCs $4 \mathrm{~h}$ after administration of LPS. Conversely, IL-10 was significantly increased in both BAL and plasma samples by delivery of MSCs. These changes are further linked to increased levels of circulating regulatory $\mathrm{T}$ cells, providing strong evidence that UC-MSCs are modifying the balance of proand anti-inflammatory agents in the immune response. Most importantly, however, these changes are met with significantly improved survival of UC-MSC treated mice. These results were further corroborated by Li et al., investigating intraperitoneally delivered LPS to induce ARDS. Similarly they found an increase in circulating levels of pro-inflammatory cytokines TNF $\alpha$, IL-1 $\beta$, and IL- 6 by $6 \mathrm{~h}$ post LPS injection, which were all significantly reduced by administration of UC-MSCs $1 \mathrm{~h}$ after treatment with LPS (Li et al. 2012). Alternatively, whereas Sun et al. found an increase in IL-10 in both BAL fluid and plasma (Sun et al. 2011), Li et al. show that treatment with LPS increases IL-10 expression, which is then unchanged by the presence of MSCs. Treatment with UC-MSCs also resulted in a modest decrease in 
immune cell infiltrate and a slight improvement to histological scores. However, most notably, animals treated with UC-MSCs, again, displayed a significant improvement in survival compared to the LPS treatment alone. Finally, in a recent study, Curley et al. (2017) investigated the idea of an off-the-shelf, xeno-free UCMSC therapy for the treatment of ARDS (Curley et al. 2017). In this study, UCMSCs were expanded under xeno-free conditions, frozen, thawed, and directly transferred into PBS for delivery into a rat, E. coli induced ARDS model. These data were also compared to BM-MSCs grown in standard xenogeneic conditions. Most notably, UC-MSCs reduced bacterial load, decreased neutrophil influx, and improved functional outcomes of lungs, compared to untreated controls, and were not different compared to BM-MSCs. Additionally, in a longitudinal study, treatment with UC-MSCs significantly improved survival of afflicted animals from $60 \%$ in the untreated group to $80 \%$ in the UC-MSC treated group. Treated animals also presented decreased TNF $\alpha$ and IL-6, and increased IL-10 within the BAL fluid, supporting the anti-inflammatory shift noted by others. However, most importantly, this study highlights the clinical potential for UC-MSCs in the treatment of ARDS. In addition to the numerous benefits associated with UC-MSCs in terms of isolation and expansion, Curley et al. provide support for their potential as an off-the-shelf, xeno-free treatment option, which is as effective in this model as BM-MSCs.

\subsection{Wound Healing and Fibrosis}

Both wound healing and development of organ fibrosis follow a cascade of processes beginning with inflammation and, unfortunately, generally ending with the formation of fibrotic scar tissue. This imperfect repair mechanism is rapid and robust, but depending on the severity of resulting fibrosis, can impact organ function. Once again, immunological effects have a critical role in the development of scar tissue, but stromal cell recruitment and activation, as well as angiogenesis are additional components involved in tissue repair.

There have been several investigations into the use of UC-MSCs, either alone or in combination with biomaterials, for treatment of wounds in animals, all showing significant positive effects of treatment. Critically, delivery of UC-MSCs into excisional wounds of immune compromised mice has been shown to significantly improve the rate of healing (Zebardast et al. 2010; Shohara et al. 2012; Fong et al. 2014). Moreover, this has been met with a more robust granulation tissue evidenced by enhanced tensile strength, increased granulation tissue thickness, increased collagen deposition, increased angiogenesis, and evidence of more mature vasculature (Zebardast et al. 2010; Shohara et al. 2012; Fong et al. 2014). Similar improvements were also noted in wounds in diabetic $\mathrm{db} / \mathrm{db}$ mice (Fong et al. 2014), and surprisingly, when either immune compromised or diabetic animals were treated with conditioned media in place of UC-MSCs, significant beneficial effects were still observed (Shohara et al. 2012; Fong et al. 2014). Addition of UC-MSCs to complement other therapeutic strategies has also been investigated. Zhang et al. looked to combine UC-MSCs with skin fragment microparticles and pieces of Wharton's Jelly, 
showing qualitatively assessed regeneration compared to scar formation in the control wounds (Zhang et al. 2012b). Combination of UC-MSCs or conditioned media with a polycaprolactone-(PCL) based scaffold incorporating aloe vera also showed evidence of regenerative effects over controls (Tam et al. 2014). The ability to add a biological component to existing biomaterials gives UC-MSCs a great potential for wound repair. The benefits of UC-MSCs are exemplified in a clinical setting where they have been applied, successfully, and without negative symptoms, to chronic wounds in dogs (Ribeiro et al. 2014). Two dogs displaying chronic wounds of duration 16 months and 24 months, both unresponsive to standard treatment, were treated with UC-MSCs delivered in poly(vinyl) alcohol (PVA) membranes. Two months following the initiation of treatment, the wounds on both dogs had re-epithelialized. The evidence provided by these studies emphasizes the pleiotropic effects of UC-MSCs on several responses involved in the repair mechanism including inflammation, granulation tissue formation, angiogenesis, and reepithelialization.

Much like in the skin, injury to internal organs requires an acute response to ameliorate damage and return function to the organ, but again this response often leads to a detrimental fibrotic repair over time. Here we will discuss studies that have investigated the use of UC-MSCs in the acute and chronic responses of organ injury.

Acute kidney injury can result from various stimuli including chemical insults, ischemia, or physical obstruction. The homeostatic response to these stimuli is often excessive, leading to further tissue damage and impaired organ function. Several studies have looked to the potent paracrine effects of UC-MSCs to shift the healing response towards a more regenerative phenotype. An initial study by Cao et al. (2010) investigating ischemia reperfusion induced acute kidney injury in immunocompetent rats showed increased tubular epithelial cell proliferation and reduced apoptosis throughout the tissue (Cao et al. 2010). Moreover, these changes were concurrent with improved renal function as measured by reduced serum creatinine and blood urea nitrogen levels. These promising results have been supported in several further studies, many of which were aimed at identifying mechanisms for these improvements. While providing benefits to the acute phase of healing, Du et al. (2012) provide additional evidence showing reduced fibrosis in UC-MSC treated groups up to 22 weeks post ischemia reperfusion injury (Du et al. 2012). In a follow up study, this reduced fibrosis was linked to a shift in growth factor production, with an increased and prolonged expression of HGF at early time points and decreased TGF $\beta 1$ expression at later time points (Du et al. 2013). While these positive results were concurrent with reduced expression of pro-inflammatory cytokines and increased expression of anti-inflammatory cytokines, other models have been used to investigate acute kidney injury showing that the positive effects are independent of cytokine levels. In an immunocompromised rat model of folic acid-induced acute kidney injury, Fang et al. (2012) investigated changes in both human-derived and rat-derived pro- and anti-inflammatory cytokines, with no differences noted between UC-MSC treated and control groups (Fang et al. 2012). The authors attribute these discrepancies with previous work to their immunocompromised model, which still showed marked functional improvements when treated with UC-MSCs. Thus, it is 
possible that the changes in cytokine expression seen in immunocompetent animals may be an artifact of the host immune response and independent of tissue injury; however, further investigation is required. In their study, Fang et al. (2012) attribute improvements to reduced apoptosis through the mitochondrial apoptotic pathway, mediated by caspase 9 . While these studies provide strong support for functional improvements, they report only very low levels of cell engraftment into the tissue, suggesting that these cells are likely acting through endocrine processes. Whereas a cytokine-mediated response is controversial, investigation into UC-MSC secreted exosomes has suggested that these may mediate UC-MSC induced functional improvements (Zhou et al. 2013b). Concentrated UC-MSC derived exosomes injected locally into the kidney yielded improvements that were not seen in UCMSC conditioned media nor in lung fibroblast derived exosome treated groups. While there is still a need to further elucidate the mechanisms of action, in a recent study Liu et al. (2016a) have expanded upon these findings by modifying UC-MSCs with retroviral delivered IGF-1. In a nude rat model of gentamicin-induced kidney injury modified UC-MSCs were shown to improve MSC homing to the injured kidney, to decrease inflammation and to reduce the amount of apoptosis over unmodified UC-MSCs. These data display hopeful results for UC-MSCs as a treatment of acute kidney injury, yet they also emphasize the critical shortcomings of rodent models, in which neither immune competent nor immune compromised animals provide a proxy for human disease. Nevertheless, these studies point in a promising direction.

Damage to the liver parenchyma via acute or chronic stimuli can lead to a potent immune response, resulting in excessive matrix production involving myofibroblastic differentiation of hepatic stellate cells (Xu et al. 2012). If the fibrotic response is not abrogated, cirrhosis can develop, characterized by architectural changes and vascular deficiency within the liver. Treatment of chemically induced liver fibrosis with UC-MSCs in animal models has shown promising results with both functional and structural improvements being noted. An initial study by Tsai et al. (2009) reports a vast decrease in collagen accumulation, and lesser myofibroblast differentiation as noted by a decreased $\alpha$ SMA content in UC-MSC treated groups (Tsai et al. 2009). These structural changes were also met with functional improvements assessed by specific enzyme levels in serum as biomarkers of hepatic health. In a time-course analysis, Lin et al. (2010) found that a reduced fibrotic score, evident following chemically induced liver fibrosis, could be detected at 14 and 21 days post UC-MSC injection, although was not evident by 7 days post injection (Lin et al. 2010). Conversely, control groups showed no improvement; however, it should be noted that no groups displayed functional improvements in the time frame investigated. These data have been further expanded by investigating dose response of UC-MSCs on treatment of liver fibrosis. Hong et al. (2014) show that an increased number of injections over time is superior for reducing collagen accumulation over larger doses delivered in a single injection (Hong et al. 2014). These initial findings have since been corroborated by additional studies investigating models of both chemically (Chai et al. 2016; Ma et al. 2016) and biologically (Hammam et al. 2016) induced liver fibrosis. 
Acute lung injury of various etiologies can lead to the development of fibroblastic foci, leading to vast structural impairments to the pulmonary parenchyma. While the development of ARDS (discussed above) can also lead to lung fibrosis, following a similar path from inflammation through to excessive matrix production, here we will focus on chemically induced acute lung injury. The first study to investigate UC-MSCs in a bleomycin-induced lung fibrosis model, by Moodley et al. 2009, showed that UC-MSCs were capable of homing specifically to fibrotic areas within the lung, whereas they were sparser in less damaged areas (Moodley et al. 2009). Moreover, UC-MSCs did not home to the lungs in control groups without bleomycin induced damage. Whole lung tissue, processed for mRNA transcript also yielded a lower expression of IFN $\gamma$, TGF $\beta 1$, MIF, TNF $\alpha$, and IL10 , suggesting a broad spectrum of regulation on both pro-fibrotic and pro-inflammatory cytokines. These changes were concurrent with reduced collagen deposition, and histological improvements which lasted until the termination of the study. There has since been limited investigation of UC-MSCs in models of lung fibrosis, with one group investigating modified UC-MSCs to improve their anti-fibrotic efficacy. Using angiotensin-converting enzyme II (ACE II) transfected UC-MSCs, Min et al. (2014) show that these modified MSCs can, in fact, reduce collagen deposition further than unmodified UC-MSCs (Min et al. 2014). Additionally, inducing apoptosis in UC-MSCs through serum starvation was shown to have a positive effect on lung fibrosis, beyond the effect of normal UC-MSCs (Liu et al. 2016c). Interestingly, the apoptotic enriched population reduced neutrophil infiltrate and reduced damage even more so than UC-MSCs alone.

In a model of chronic pancreatitis induced by dibutyltin dichloride (DBTC), Zhou et al. (2013a) investigated the effect of intravenous delivery of UC-MSCs. Homing of UC-MSCs to the pancreas of injured animals was noted at 14 days and 28 days post injection, but was not noted in uninjured animals injected with cells (Zhou et al. 2013a). Importantly, an investigation into the biodistribution of UC-MSCs in several organs yielded an inability to detect UC-MSCs within the lungs or kidneys of injured, injected mice; however, there was accumulation within the liver, which importantly also showed signs of injury from the DBTC injection. Conversely, in the uninjured group, some UC-MSCs had accumulated in the lungs, but were not seen in the kidney or liver. Critically, reduced immune cell infiltrate and reduced fibrosis were also seen in animals treated with UC-MSCs, correlating with improved functional outcomes in these animals.

\subsection{Bone and Joint Repair}

Much like their role in repair of soft tissues, UC-MSCs have been shown to improve healing of bone defects. Todeschi et al. (2015), investigated both UCMSCs and BM-MSCs in the repair of calvarial bone in mice, and while both improved repair to a similar extent, the mechanisms were distinct (Todeschi et al. 2015). Initially when implanted subcutaneously on a ceramic scaffold, BMMSCs formed ectopic bone of human origin whereas UC-MSCs only induced a 
dense fibrous tissue, of mouse origin. This is in comparison to the scaffold alone which induced formation of a looser fibrous tissue. Still, when either cell was applied to a calvarial defect, bone regeneration was significantly improved over the scaffold alone and was not different between BM-MSCs or UC-MSCs. Moreover, whereas human-derived cells could be identified to have differentiated into the bone in BM-MSC treated defects, this was not evident in the UC-MSC treated groups. Thus, while both treatment options led to improvements to bone regeneration, BM-MSCs directly contributed to bone formation whereas UC-MSCs acted only through paracrine effects. In two additional studies, directed differentiation studies were carried out in vitro prior to assessment of MSCs in vivo. Investigating induced pluripotent stem cells (iPSCs), BM-MSCs, and UC-MSCs in the repair of a damaged calvarial bone of rats, Wang et al. (2015) first showed differences in directed bone differentiation between different MSC origins (Wang et al. 2015). Importantly, while there were moderate differences in the extent of bone differentiation in vitro, with iPSCs tending to show decreased differentiation, all celltreated groups performed equally in vivo. All cells, delivered on a calcium phosphate-based biomaterial, formed dense bone, and induced angiogenesis, to equal levels which were not observed in the biomaterial only control group. In fact, the only difference between cell-treated groups was that iPSCs stimulated slightly better integration with the native bone. Similarly, Kajiyama et al. (2015) found in their cell populations that UC-MSCs tended to underperform in bonedirected differentiation assays compared to BM-MSCs (Kajiyama et al. 2015). Conversely, in vivo only UC-MSCs significantly increased the bone volume of calvarial defects, whereas BM-MSCs had an intermediate effect, which was not different from the untreated control. These discrepancies between in vitro differentiation and in vivo performance reported by Wang et al. and Kajiyama et al. stress the importance of identifying meaningful potency assays for optimal cell selection for a given indication (Ujiie et al. 2015). Moreover, as paracrine effects, and not direct differentiation, are most often cited as the therapeutically relevant contribution of MSCs, analysis of differentiation as outlined in the ISCT's minimal criteria for defining MSCs (Dominici et al. 2006), likely does not relate to a population's ability to contribute to any malady in vivo.

UC-MSCs have also shown utility in the treatment of tendon injury. In a model of collagenase induced injury of the Achilles tendon in nude rats, Emrani and Davies (2011) show that injected UC-MSCs incorporated into the injured tendon and increase the mRNA expression of type I collagen within the tissue (Emrani 2011). Furthermore, mechanical testing of the tendon 30 days after injury revealed that UCMSC treatment improved tensile strength from $41 \%$ of uninjured tendon in the control group up to $68 \%$ of uninjured tendon.

To our knowledge, human UC-MSCs have only been investigated in one study for the treatment of osteoarthritis (Saulnier et al. 2015). In a rabbit model of medial meniscal release induced osteoarthritis, UC-MSCs were injected at either 3 or 15 days following induction and rabbits were examined for gross morphological, histological, and gene expression changes. Only modest visual improvement was 
seen by 56 days post injury in groups treated with UC-MSCs on day 3 post injury. Surprisingly, inflammatory cell infiltrate was only seen in UC-MSC injected animals and persisted until day 56 which is possibly mediated by the upregulation of both proinflammatory TNF $\alpha$ and anti-inflammatory IL-10 by UC-MSCs. Still, only injection of UC-MSCs at the earlier time point led to the upregulation of COL2a in the cartilage by 56 days, suggesting that cartilage repair and remodeling may be supported by injection of UC-MSCs.

\subsection{Ischemia}

Unilateral femoral artery ligation to promote hind limb ischemia in rodents is a widely used model to study critical limb ischemia. Grounded on the pro-angiogenic nature of MSCs, strategies to promote the growth of neovasculature to mitigate ischemia using these cells and their secreted products have been developed. Zhang et al. (2012a) first showed that UC-MSCs could be used to enhance angiogenesis and improve circulation within the ischemic hind limb, through a mechanism mediated by the release of microvesicles. In fact, concentrated microvesicles isolated from culture media could reestablish circulation within the ligated vasculature to the same extent as injected UC-MSCs. Others have expanded upon these initial findings by modifying UC-MSCs in culture to improve recovery of blood flow following ligation. Shen et al. (2013) investigated the use of endothelial progenitor cells, derived from UC-MSCs, to improve hind limb ischemia (Shen et al. 2013). These cells enhanced angiogenesis, and decreased both muscle degeneration and the extent of apoptosis in the ischemic tissue. Critically, these changes were sufficient to improve overall blood flow within the limb and improve functional recovery of the limb. Finally, Han et al. (2016) have shown that hypoxia preconditioned UCMSCs injected into the hypoxic muscle tissue following femoral artery ligation, enhance the pro-angiogenic effect of UC-MSCs grown in normoxic conditions, increasing both the capillary density and the ratio of capillaries to muscle fibers (Han et al. 2016).

\subsection{Myocardial Infarction}

Temporary ligation of the left anterior descending (LAD) branch of the coronary artery is a common method to induce myocardial infarction in animal models, creating an ischemic area near the apex of the heart. Instead of a regenerative process to replace the damaged cardiomyocytes, a cascade of events follows such an ischemic episode that leads to the formation of scar tissue, impairing organ function. Initially, Dayan et al. (2011) investigated how MSC-induced changes in the immune response following myocardial infarction could modify outcomes in mice (Dayan et al. 2011). Both UCMSCs and BM-MSCs, delivered intravenously, decreased total number of circulating 
macrophages following injury, with BM-MSCs also decreasing macrophage localization in the heart. Furthermore, the macrophages present showed greater polarization to the M2 anti-inflammatory phenotype, which the authors link to paracrine secretion of IL-10 evident in animals treated by either UC-MSC or BM-MSCs. MSC treatment also led to a decrease in the number of apoptotic cardiomyocytes, yet did not induce an angiogenic response. Only a minor improvement to fractional shortening was evident at early timepoints in animals treated with MSCs, but this was not significantly different to control animals by 16 weeks post injury. Other functional parameters were not different between groups. Critically, although overall health of the animals treated with MSCs was slightly improved as evident by decreased lung congestion, no improvements to survival were seen (Dayan et al. 2011). Thus, while MSCs could modify the initial innate immune response, this alone was insufficient to improve outcomes in this model of myocardial infarction. To expand upon these data, Yannarelli et al. (2013) investigated an alternative MSC delivery strategy via intramyocardial injection (Yannarelli et al. 2013). Whereas intravenous delivery of UC-MSCs and BM-MSCs led primarily to localization within the lungs and did not affect cardiac function, local delivery of MSCs significantly improved fractional shortening, with UC-MSCs providing a superior response over BM-MSCs. Conversely, neither septum thickness nor other gross structural parameters were altered by MSC treatment. While neither MSC-treated groups displayed altered scar tissue formation, only treatment with UC-MSCs enhanced the angiogenic response over the control group (Yannarelli et al. 2013). Santos Nascimento et al. (2014) have corroborated these data, finding that treatment with UC-MSCs, delivered through intramyocardial injection, decreased the number of apoptotic cells, and modestly increased CD31+ cell infiltrate in the infarcted region, but no functional improvements, including fractional shortening, were resolved (Santos Nascimento et al. 2014). Further, in a miniswine model of myocardial infarction, Zhang et al. (2013) observed moderate functional and structural improvements following intramyocardial delivery of UC-MSCs (Zhang et al. 2013b). These were associated with greatly improved angiogenesis and a reduced number of apoptotic cells. Additionally, quantification of cell marker expression suggested that UC-MSCs induced infiltration and activation of cardiac stem cells in the ischemic tissue, potentially leading to a more regenerative phenotype. Consequently, these investigations into the utility of UCMSCs for the treatment of acute myocardial infarction have yielded variable results. While UC-MSC treated groups display positive effects at the cellular level, these are not always met with functional improvements, suggesting that although they may ameliorate specific aspects of the natural response, they may be more beneficial when combined with other treatment modalities. Conversely, a recent study by Liu et al. (2016), investigating chronic myocardial ischemia in a porcine model, shows promise for UC-MSCs in this indication (Liu et al. 2016b). Four weeks prior to UC-MSC delivery, an ameroid constrictor was placed in the left coronary artery to restrict blood flow. In the follow up period, which lasted an additional four weeks after cell delivery, pigs receiving UC-MSCs displayed improvements in a range of functional parameters, including decreased heart rates, and improved ejection fraction and fractional shortening (Liu et al. 2016b). Moreover, treatment with UC-MSCs resulted in an improved angiogenic response, decreased apoptosis, and decreased fibrosis. 


\subsection{Diabetes}

Although several secondary maladies can develop over time in diabetic patients, here we will focus solely on the aspects of insulin-mediated control of blood glucose. Several studies have investigated the use of pancreatic $\beta$-like cells derived from UCMSC progenitors to treat chemically induced type 1 diabetes (Kadam and Bhonde 2010; Tsai et al. 2012; Wang et al. 2014b). In all cases the differentiation protocols used resulted in a significantly, and greatly increased expression of both insulin and C-peptide, suggesting a $\beta$-like population. In these studies, UC-MSC-derived cells were delivered intravenously (Tsai et al. 2012), within a polymer capsule (Kadam and Bhonde 2010), or into the renal capsule (Wang et al. 2014b), and in all cases a reduced resting blood glucose was noted compared to untreated animals with induced diabetes. Although Kadam and Bhonde (2010) reported improvements equal to the level of non-diabetic animals, both Tsai et al. (2012) and Wang et al. (2014c) showed an intermediate response that remained worse than non-diabetic animals.

Type 2 diabetes has been approached in two studies investigating different aspects of the disease. Hu et al. (2014) first investigated a high fat diet, low dose streptozotocin induced type 2 mimicking regime in rats (Hu et al. 2014). In their model, treatment with UC-MSCs in combination with sitagliptin, an anti-diabetic drug targeting dipeptidyl peptidase 4 , decreased fasting plasma glucose to the level of control, non-diabetic mice, an improvement over either treatment individually. Furthermore, they displayed recovery and regeneration of depleted pancreatic $\beta$-cells, a response also evident in UC-MSCs alone. Still, the mechanisms through which these two treatment modalities interact is uncertain. While regeneration of $\beta$-cells is a key component of type 2 diabetes treatment, insulin sensitization, especially in adipose tissue is equally important. Using the same model of type 2 diabetes, Xie et al. (2016) investigated the effect of UC-MSC delivery on the inflammatory state of endogenous adipose tissue to recover insulin sensitivity (Xie et al. 2016). Intravenous delivery of UC-MSCs significantly reduced resting blood glucose, in addition to improving glucose lowering following challenge via an intraperitoneal glucose tolerance test, although in both cases, not to the extent of non-diabetic animals. UC-MSC treated animals also displayed a decreased insulin resistance as measured by the product of the resting blood glucose and resting blood insulin levels compared to untreated diabetic animals. These changes were concomitant with greatly increased expression of anti-inflammatory M2 macrophage markers within the adipose tissue, and a decreased expression of pro-inflammatory M1 macrophage markers. Thus, it seems that treatment of type 2 diabetes with systemic dosing of UC-MSCs provides a twopronged approach targeting both insulin resistance and insulin production through $\beta$-cell regeneration, indicating promise for future studies. 


\subsection{Cancer}

Human cancer xenografts offer a unique tool to study relevant cancer cell growth kinetics under various pressures in a living model system. Using the MDA-231 breast cancer cell line, tumors rapidly develop in the lungs of SCID mice following delivery via tail vein injection (Ayuzawa et al. 2009). However, UC-MSCs injected weekly starting 8 days after MDA-231 cells, homed to tumors within the lungs and significantly attenuated tumor growth following 3 weekly UC-MSC injections. In a second study, Wu et al. (2013) provide evidence that microvesicles released from UC-MSCs may be mediating this response (Wu et al. 2013). Using a T24 bladder cancer cell xenograft in nude mice, UC-MSCs or UC-MSC-derived microvesicles, injected alongside T24 cells, both significantly reduced tumor growth by 30 days, yet the concentrated microvesicles outperformed UC-MSCs. In both treatment groups, decreased cell proliferation and increased apoptosis within the tumors were noted. Still, the mechanisms through which the microvesicles are functioning in this system are unknown. It should also be noted that UC-MSCs have been shown to enhance tumor development, growth and metastasis in xenograft models. In an esophageal carcinoma cell line, Eca109, UC-MSCs increased tumor growth when injected simultaneously to cancer cells in a nude mouse xenograft model (Yang et al. 2014). Furthermore, when injected after tumors had already been established, enhanced tumor growth and metastasis to the lymph nodes were observed in UCMSC treated groups. It is possible that there are type-specific interactions between cancer cells and UC-MSCs, and thus there is a need for investigation into these responses. Conversely, an alternative approach has recently been investigated for the use of UC-MSCs as therapeutics in cancer treatment. Using the natural homing nature of UC-MSCs Yan et al. (2016) have modified these cells to produce a soluble TRAIL ligand to induce apoptosis in established lung tumors of A549 lung cancer cell xenografts (Yan et al. 2016). Although the level of apoptosis within the tumor was greatly increased by the modified UC-MSCs, no difference in tumor size or animal survival were noted compared to the control groups. However, this approach permits the possibility to include additional modifications to improve upon these effects moving forward.

\subsection{Antibody Therapy and Biodefense}

The immune-privileged nature of UC-MSCs, allowing them to persist in immune competent allogeneic hosts for unprecedented duration, offers a unique system for delivery of biological payloads. Recently, Braid et al. (2016) published data in immune-compromised mice showing that the delivery and release of a prophylactic antibody could be delivered by transfected UC-MSCs, with the antibody remaining at a predicted protective level for up to 38 days following delivery and above the detectable limit for up to 109 days (Braid et al. 2016). In this study, an antiVenezuelan equine encephalitis virus (VEEV) antibody delivered via transduced UC-MSCs into mice 10-days or $24 \mathrm{~h}$ prior to exposure to lethal doses of VEEV, 
greatly improved survival of animals, and reduced disease score in those animals exposed. These improvements were noted compared to untreated controls as well as controls treated with purified anti-VEEV antibody. These exciting data hold great promise for a prophylactic therapy that could have implications in biodefense or disease outbreaks. Additionally, this technology could be used to treat patients requiring antibody-based therapies to provide prolonged delivery of clinically relevant doses of monoclonal antibodies.

\section{UC MSCs as Therapeutics for Human Disease}

As of January 2016, there were 109 clinical trials registered on the ClinicalTrials.gov database involving umbilical cord tissue cells either alone or in conjunction with another cell type, but excluding those employing solely umbilical cord blood derived cells. These include Phase 1, 2, and 3 trials and therefore the current number should more accurately be recorded as 68. Of these 53 are in China, and the rest in Panama (6), Korea (2), Turkey (2), Chile (1), Italy (1), Indonesia (1), Taiwan (1), and the USA (1). Registration in this database is voluntary and does not include all clinical trials involving umbilical cord derived cells, although we restrict our analysis here to registered trials.

The trials have been targeted at 14 broad groups of medical indications, as illustrated in Fig. 5. The preponderance of trials has targeted autoimmune, cardiovascular, hematological, hepatic, neurodegenerative, and orthopedic conditions, each of which commands approximately $10 \%$ of the total number. The first trial [NCT00951210] targeted autism. This and four other trials were initiated in China, in 2009, for liver cirrhosis, liver failure, systemic sclerosis, and Type1 diabetes. Examples of trials in subsequent years illustrate the increasing breadth of conditions targeted. Thus, three new liver cirrhosis trials, and another T1 diabetes trial, were added in 2010 among others that included broncopulmonary dysplasia and ulcerative colitis. 2011 saw enrollment in the first trials for stroke and Alzheimer's disease, in addition to acute myocardial infarction and T2 Diabetes. 2012 saw the first GvHD trial and co-administration with organ transplants, and two new trials targeting lupus. Both osteoarthritis and rheumatoid arthritis trials were added in 2013 together with MS and fracture non-union while 2015 saw three trials targeted at lung pathologies.

Since, the ClinicalTrials.gov data contains no details of the cell extraction methods employed it is not possible to compare the individual therapeutic products. However, some information is available in related publications. NCT01343511 reported an increased therapeutic effect of UCMSCs over cord blood mononuclear cells alone (Lv et al. 2013); NCT01360164 confirmed safety and a possible delay in the progression of spinocerebellar ataxia (Jin et al. 2013); NCT01741857 reported a satisfactory response in systemic lupus erythematosus (Wang et al. 2014a; Wang et al. 2014b); NCT01213186 reported improved host immune reconstitution in immune non-responders (Zhang et al. 2013a); and NCT01547091 showed that administration of umbilical cord cells with diseasemodifying anti-rheumatic drugs provided persistent clinical benefits for patients 


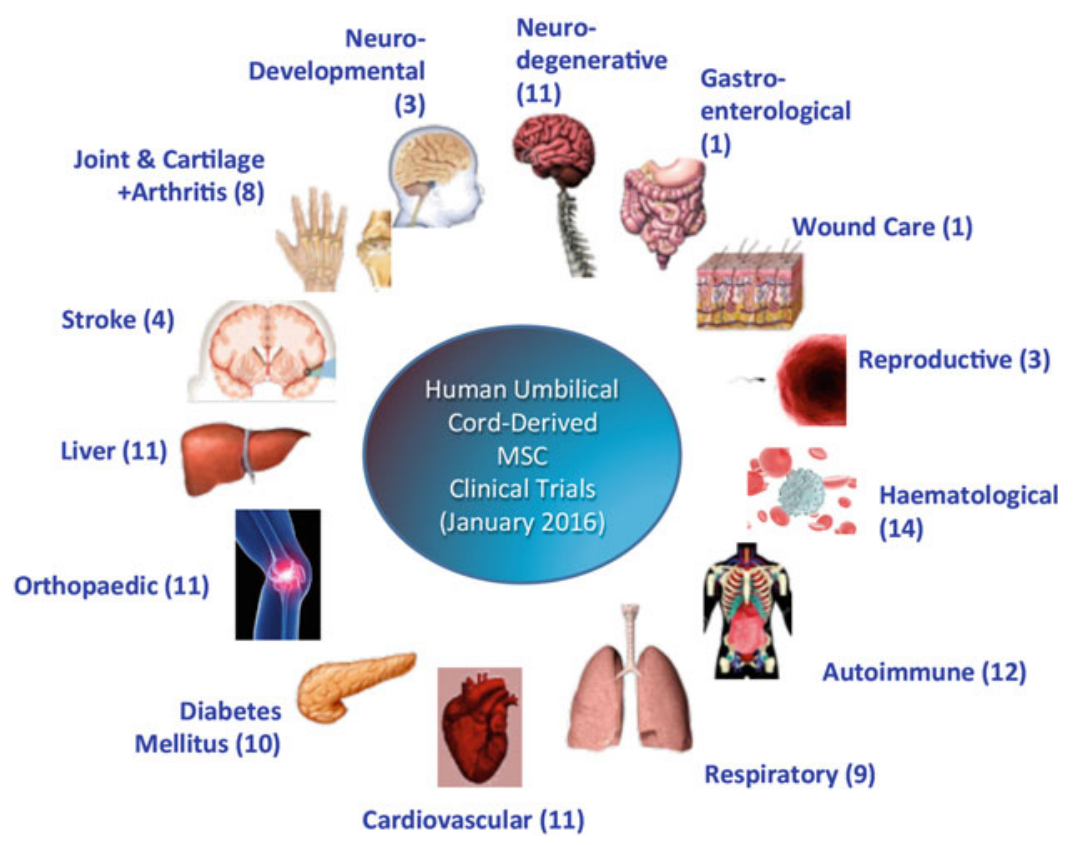

Fig. 5 The pictogram represents the range of clinical trials registered on ClinicalTrials.gov and employing human umbilical cord tissue derived MSC (excluding those from cord blood). This distribution is broken down in the pie-chart as percentages of the total per indication group. (Data courtesy of Tissue Regeneration Therapeutics Inc.)

with rheumatoid arthritis (Wang et al. 2013). It should be noted that none of these small studies was blinded, none were randomized and only some were controlled. Furthermore, while all studies used an intravenous route of administration, two also employed intrathecal delivery. The I.V. dose varied from $0.5 \times 10^{6}$ to $4 \times$ $10^{7} \mathrm{cells} / \mathrm{Kg}$ body weight. Taken together, these studies do point to the administration of umbilical cord cells being safe and possibly providing some therapeutic benefit, although definitive information will not be available until large scale randomized, double blinded, placebo-controlled trials are undertaken.

\section{Conclusions}

If reaching clinical trials is the coming of age of a new cell therapeutic approach, then UC-MSCs can be considered to have reached this significant milestone. However, it remains to be seen - as with most attempts to employ MSCs from any tissue source - if robust and positive clinical outcomes can be achieved. Inevitably, progress in the employment of umbilical cord-derived mesenchymal cells is hampered by the lack of consensus on both anatomical descriptors of the cord tissue and 
standardization of cell isolation techniques. To promote progress, we consider it essential that both preclinical and clinical papers and reports provide sufficient methodological detail to allow repetition of the work by others in the field. This, after all, is considered the bedrock of good scientific reporting but, as we have shown herein, is lacking with respect to this increasingly important source of cells. Nevertheless, we remain optimistic that umbilical cord-derived mesenchymal stromal cells have great potential in providing therapeutic solutions for a large number of clinical indications.

\section{References}

Agah ME, Parivar K, Nabiuni M, Hashemi M, Soleimani M (2013) Induction of human umbilical Wharton's jelly-derived stem cells toward oligodendrocyte phenotype. J Mol Neurosci 51:328-336

Ayuzawa R, Doi C, Rachakatla RS, Pyle MM, Maurya DK, Troyer D, Tamura M (2009) Naïve human umbilical cord matrix derived stem cells significantly attenuate growth of human breast cancer cells in vitro and in vivo. Cancer Lett 280:31-37

Bosch J, Houben AP, Radke TF, Stapelkamp D, Bunemann E, Balan P, Buchheiser A, Liedtke S, Kögler G (2012) Distinct differentiation potential of "MSC" derived from cord blood and umbilical cord: are cord-derived cells true mesenchymal stromal cells? Stem Cells Dev 21:1977-88

Bouma G, Strober W (2003) The immunological and genetic basis of inflammatory bowel disease. Nat Rev Immunol 3:521-533

Braid LR, Hu W-G, Davies JE, Nagata LP (2016) Engineered mesenchymal cells improve passive immune protection against lethal venezuelan equine encephalitis virus exposure. Stem Cels Transl Med 5:1-10

Can A, Karahuseyinoglu S (2007) Concise review: human umbilical cord stroma with regard to the source of fetus-derived stem cells. Stem Cells 25:2886-2895

Cao H, Qian H, Xu W, Zhu W, Zhang X, Chen Y, Wang M, Yan Y, Xie Y (2010) Mesenchymal stem cells derived from human umbilical cord ameliorate ischemia/reperfusion-induced acute renal failure in rats. Biotechnol Lett 32:725-732

Caplan AI (2007) Adult mesenchymal stem cells for tissue engineering versus regenerative medicine. J Cell Physiol 213:341-347

Chai N-L, Zhang X-B, Chen S-W, Fan K-X, Linghu E-Q (2016) Umbilical cord-derived mesenchymal stem cells alleviate liver fibrosis in rats. World J Gastroenterol 22:6036

Chao YH, Wu HP, Wu KH, Tsai YG, Peng CT, Lin KC, Chao WR, Lee MS, Fu YC (2014) An increase in $\mathrm{CD} 3+\mathrm{CD} 4+\mathrm{CD} 25+$ regulatory $\mathrm{T}$ cells after administration of umbilical cord-derived mesenchymal stem cells during sepsis. PLoS One 9:1-8

Chen Y, Song Y, Miao H, Xu Y, Lv M, Wang T, Hou Y (2015) Gene delivery with IFN-gammaexpression plasmids enhances the therapeutic effects of MSCs on DSS-induced mouse colitis. Inflamm Res 64:671-681

Cohen J (2002) The immunopathogenesis of sepsis. Nature 420:885-891

Coskun H, Can A (2015) The assessment of the in vivo to in vitro cellular transition of human umbilical cord multipotent stromal cells. Placenta 36:232-239

Curley GF, Jerkic M, Dixon S, Hogan G, Masterson C, O’Toole D, Devaney J, Laffey JG (2017) Cryopreserved, xeno-free human umbilical cord mesenchymal stromal cells reduce lung injury severity and bacterial burden in rodent. Escherichia coli-induced acute respiratory distress syndrome. Crit Care Med. 45:e202-e212

Davies JE, Walker JT, Keating AK (2017) Wharton's jelly: the rich, but enigmatic, source of MSCs. Stem Cells Transl Med 6(7):1620-1630 
Dayan V, Yannarelli G, Billia F, Filomeno P, Wang XH, Davies JE, Keating A (2011) Mesenchymal stromal cells mediate a switch to alternatively activated monocytes/macrophages after acute myocardial infarction. Basic Res Cardiol 106:1299-1310

Dominici M, Le Blanc K, Mueller I, Slaper-Cortenbach I, Marini F, Krause D, Deans R, Keating A, Prockop D, Horwitz E (2006) Minimal criteria for defining multipotent mesenchymal stromal cells. The international society for cellular therapy position statement. Cytotherapy 8:315-317

Donders R, Vanheusden M, Bogie JFJ, Ravanidis S, Thewissen K, Stinissen P, Gyselaers W, Hendriks JJA, Hellings N (2015) Human Wharton's jelly-derived stem cells display immunomodulatory properties and transiently improve rat experimental autoimmune encephalomyelitis. Cell Transplant 24:2077-2098

Du T, Cheng J, Zhong L, Zhao X, Zhu J, Zhu Y-J, Liu G-H (2012) The alleviation of acute and chronic kidney injury by human Wharton's jelly-derived mesenchymal stromal cells triggered by ischemia-reperfusion injury via an endocrine mechanism. Cytotherapy 14:1215-1227

Du T, Zou X, Cheng J, Wu S, Zhong L, Ju G, Zhu J, Liu G (2013) Human Wharton 's jelly-derived mesenchymal stromal cells reduce renal fibrosis through induction of native and foreign hepatocyte growth factor synthesis in injured tubular epithelial cells. Stem Cell Res Ther 4:1

Emrani H, Davies JE (2011) Umbilical cord perivascular cells: A mesenchymal cell source for treatment of tendon injuries. Open Tiss Eng Regen Med J 4:112-119

Ennis J, Götherström C, Le Blanc K, Davies JE (2008) In vitro immunologic properties of human umbilical cord perivascular cells. Cytotherapy 10:174-181

Fan H, Zhao G, Liu L, Liu F, Gong W, Liu X, Yang L, Wang J, Hou Y (2012) Pre-treatment with IL$1 \beta$ enhances the efficacy of MSC transplantation in DSS-induced colitis. Cell Mol Immunol 9:473-481

Fang TC, Pang CY, Chiu SC, Ding DC, Tsai RK (2012) Renoprotective effect of human umbilical cord-derived mesenchymal stem cells in immunodeficient mice suffering from acute kidney injury. PLoS One 7:1-15

Farias VA, Linares-Fernández JL, Peñalver JL, Payá Colmenero JA, Ferrón GO, Duran EL, Fernández RM, Olivares EG, O’Valle F, Puertas A, Oliver FJ, Ruiz De Almodóvar JM (2011) Human umbilical cord stromal stem cell express CD10 and exert contractile properties. Placenta 32:86-95

Fong CY, Subramanian A, Biswas A, Gauthaman K, Srikanth P, Hande MP, Bongso A (2010) Derivation efficiency, vcell proliferation, freeze-thaw survival, stem-cell properties and differentiation of human Wharton's jelly stem cells. Reprod Biomed Online 21:391-401

Fong CY, Tam K, Cheyyatraivendran S, Gan SU, Gauthaman K, Armugam A, Jeyaseelan K, Choolani M, Biswas A, Bongso A (2014) Human Wharton's Jelly stem cells and its conditioned medium enhance healing of excisional and diabetic wounds. J Cell Biochem 115:290-302

Hammam OA, Elkhafif N, Attia YM, Mansour MT, Elmazar MM, Abdelsalam RM, Kenawy SA, El-Khatib AS (2016) Wharton's jelly-derived mesenchymal stem cells combined with praziquantel as a potential therapy for Schistosoma mansoni-induced liver fibrosis. Sci Rep 6:21005

Han KH, Kim AK, Kim MH, Kim DH, Go HN, Kim DI (2016) Enhancement of angiogenic effects by hypoxia-preconditioned human umbilical cord-derived mesenchymal stem cells in a mouse model of hindlimb ischemia. Cell Biol Int 40:27-35

Hong J, Jin H, Han J, Hu H, Liu J, Li L, Huang Y, Wang D, Wu M, Qiu L, Qian Q (2014) Infusion of human umbilical cord-derived mesenchymal stem cells effectively relieves liver cirrhosis in DEN-induced rats. Mol Med Rep 9:1103-1111

Hu J, Wang F, Sun R, Wang Z, Yu X, Wang L, Gao H, Zhao W, Yan S, Wang Y (2014) Effect of combined therapy of human Wharton's jelly-derived mesenchymal stem cells from umbilical cord with sitagliptin in type 2 diabetic rats. Endocrine 45:279-287

Ilancheran S, Michalska A, Peh G, Wallace EM, Pera M, Manuelpillai U (2007) Stem Cells Derived from Human Fetal Membranes Display Multilineage Differentiation Potential. Biol Reprod 77(3):577-588 
Jin J, Liu Z, Lu Z, Guan D, Wang C, Chen Z, Zhang W, Wu J, Xu Y (2013) Safety and efficacy of umbilical cord mesenchymal stem cell therapy in hereditary spinocerebellar ataxia. Curr Neurovasc Res 10:11-20

Kadam SS, Bhonde RR (2010) Islet neogenesis from the constitutively nestin expressing human umbilical cord matrix derived mesenchymal stem cells. Islets 2:112-120

Kajiyama S, Ujiie Y, Nishikawa S, Inoue K, Shirakawa S, Hanada N, Liddell R, Davies JE, Gomi K (2015) Bone formation by human umbilical cord perivascular cells. J Biomed Mater Res Part A 103:2807-2814

Kim WY, Hong SB (2016) Sepsis and acute respiratory distress syndrome: recent update. Tuberc Respir Dis 79:53-57

Kita K, Gauglitz GG, Phan TT, Herndon DN, Jeschke MG (2010) Isolation and characterization of mesenchymal stem cells from the sub-amniotic human umbilical cord lining membrane. Stem Cells Dev 19:491-502

Li J, Li D, Liu X, Tang S, Wei F (2012) Human umbilical cord mesenchymal stem cells reduce systemic inflammation and attenuate LPS-induced acute lung injury in rats. J Inflamm 9:33

Liang L, Dong C, Chen X, Fang Z, Xu J, Liu M, Zhang X, Gu DS, Wang D, Du W, Zhu D, Han Z (2011) Human umbilical cord mesenchymal stem cells ameliorate mice TNBS-induced colitis. Cell Transplant 20:1395-1408

Lin S-Z, Chang Y-J, Liu J-W, Chang L-F, Sun L-Y, Li Y-S, Luo G-H, Liao C-H, Chen P-H, Chen TM, Lee R-P, Yang K-L, Harn H-J, Chiou T-W (2010) Transplantation of human Wharton's Jellyderived stem cells alleviates chemically induced liver fibrosis in rats. Cell Transplant 19:1451-1463

Lin Y, Lin L, Wang Q, Jin Y, Zhang Y, Cao Y, Zheng C (2015) Transplantation of human umbilical mesenchymal stem cells attenuates dextran sulfate sodium-induced colitis in mice. Clin Exp Pharmacol Physiol 42:76-86

Liu R, Zhang Z, Lu Z, Borlongan C, Pan J, Chen J, Qian L, Liu Z, Zhu L, Zhang J, Xu Y (2013) Human umbilical cord stem cells ameliorate experimental autoimmune encephalomyelitis by regulating immunoinflammation and remyelination. Stem Cells Dev 22:1053-1062

Liu P, Feng Y, Dong D, Liu X, Chen Y, Wang Y, Zhou Y (2016a) Enhanced renoprotective effect of IGF-1 modified human umbilical cord-derived mesenchymal stem cells on gentamicin-induced acute kidney injury. Sci Rep 6:20287

Liu C-B, Huang H, Sun P, Ma S-Z, Liu A-H, Xue J, Fu J-H, Liang Y-Q, Liu B, Wu D-Y, Lu S-H, Zhang X-Z (2016b) Human umbilical cord-derived mesenchymal stromal cells improve left ventricular function, perfusion, and remodeling in a porcine model of chronic myocardial ischemia. Stem Cells Transl Med 5:1004-1013

Liu F-B, Lin Q, Liu Z-W, Liu Z (2016c) A study on the role of apoptotic human umbilical cord mesenchymal stem cells in bleomycin-induced acute lung injury in rat models. Eur Rev Med Pharmacol Sci 20:969-982

Lu L, Zhao Q, Wang X, Xu Z, Lu Y, Chen Z, Liu Y (2006) Isolation and characterization of human umbilical cord mesenchymal stem cells with hematopoiesis-supportive function and other potentials. Haematologica 91:1017-1026

Lv Y, Zhang Y, Liu M, Qiuwaxi J, Ashwood P, Cho SC, Huan Y, Ge R, Chen X, Wang Z, Kim B, Hu X (2013) Transplantation of human cord blood mononuclear cells and umbilical cord-derived mesenchymal stem cells in autism. J Transl Med 11:196

Ma J, Li L, Chen L, Wang X (2016) Effects of human umbilical cord mesenchymal stem cells in the treatment of CCl4-induced liver cirrhosis. Int J Clin Exp Med 9:5168-5180

Machuca TN, Cypel M (2014) Ex vivo lung perfusion. J Thoracic Disease 6:1054-1062

Maruyama Y (1963) The human endothelial cell in tissue culture. Zeitschrift fiir Zellforschung 60:69-79

Masterson C, Jerkic M, Curley GF, Laffey JG (2015) Mesenchymal stromal cell therapies: potential and pitfalls for ARDS. Minerva Anestesiol 81:179-194

McQualter JL, Bernard CCA (2007) Multiple sclerosis: a battle between destruction and repair. J Neurochem 100:295-306 
Min F, Gao F, Li Q, Liu Z (2014) Therapeutic effect of human umbilical cord mesenchymal stem cells modified by angiotensin-converting enzyme 2 gene on bleomycin-induced lung fibrosis injury. Mol Med Rep 11:2387-2396

Moodley Y, Atienza D, Manuelpillai U, Samuel CS, Tchongue J, Ilancheran S, Boyd R, Trounson A (2009) Human umbilical cord mesenchymal stem cells reduce fibrosis of bleomycin-induced lung injury. Am J Pathol 175:303-313

Mordant P, Nakajima D, Kalaf R, Iskender I, Maahs L, Behrens P, Coutinho R, Iyer RK, Davies JE, Cypel M, Liu M, Waddell TK, Keshavjee S (2016) Mesenchymal stem cell treatment is associated with decreased perfusate concentration of IL-8 during ex vivo perfusion of donor lungs after $18 \mathrm{~h}$ preservation. J Heart Lung Transplant 35:1245-1254

Nanaev AK, Kohnen G, Milovanov AP, Domogatsky SP, Kaufmann P (1997) Stromal differentiation and architecture of the human umbilical cord. Placenta 18(1):53-64

Payne NL, Sun G, Mcdonald C, Layton D, Moussa L, Emerson-Webber A, Veron N, Siatskas C, Herszfeld D, Price J, Bernard CCA (2013) Distinct immunomodulatory and migratory mechanisms underpin the therapeutic potential of human mesenchymal stem cells in autoimmune demyelination. Cell Transplant 22:1409-1425

Ribeiro J, Pereira T, Amorim I, Caseiro AR, Lopes MA, Lima J, Gartner A, Santos JD, Bártolo PJ, Rodrigues JM, Mauricio AC, Luís AL (2014) Cell therapy with human MSCs isolated from the umbilical cord Wharton Jelly associated to a PVA membrane in the treatment of chronic skin wounds. Int J Med Sci 11:979-987

Santos Nascimento D, Mosqueira D, Sousa LM, Teixeira M, Filipe M, Resende TP, Araújo AF, Valente M, Almeida J, Martins JP, Santos JM, Bárcia RN, Cruz P, Cruz H, Pinto-do-Ó P (2014) Human umbilical cord tissue-derived mesenchymal stromal cells attenuate remodeling after myocardial infarction by proangiogenic, antiapoptotic, and endogenous cell-activation mechanisms. Stem Cell Res Ther 5:5

Sarugaser R, Lickorish D, Baksh D, Hosseini M, Davies JE (2005) Human Umbilical Cord Perivascular (HUCPV) Cells: A Source of Mesenchymal Progenitors. Stem Cells 23(2): 220-229

Sarugaser R, Hanoun L, Keating A, Stanford WL, Davies JE (2009) Human mesenchymal stem cells self-renew and differentiate according to a deterministic hierarchy. PLoS One 4:e6498

Saulnier N, Viguier E, Perrier-groult E, Chenu C, Pillet E, Roger T, Maddens S, Boulocher C (2015) Intra-articular administration of xenogeneic neonatal Mesenchymal Stromal Cells early after meniscal injury down-regulates metalloproteinase gene expression in synovium and prevents cartilage degradation in a rabbit model of osteoarthritis. Osteoarthr Cartil 23:122-133

Schugar RC, Chirieleison SM, Wescoe KE, Schmidt BT, Askew Y, Nance JJ, Evron JM, Peault B, Deasy BM (2009) High harvest yield, high expansion, and phenotype stability of CD146 mesenchymal stromal cells from whole primitive human umbilical cord tissue. J Biomed Biotechnol 2009:789526

Shen W, Liang C, Wu V-C, Wang S-H, Young G, Lai I, Chien C, Wang S, Wu K, Chen Y (2013) Endothelial progenitor cells derived from Wharton's Jelly of the umbilical cord reduces ischemia-induced hind limb injury in diabetic mice by inducing HIF-1 $\alpha /$ IL-8 expression. Stem Cells Dev 22:1408-1418

Shohara R, Yamamoto A, Takikawa S, Iwase A, Hibi H, Kikkawa F, Ueda M (2012) Mesenchymal stromal cells of human umbilical cord Wharton's Jelly accelerate wound healing by paracrine mechanisms. Cytotherapy 14:1171-1181

Steinman L (2014) Immunology of relapse and remission in multiple sclerosis. Annu Rev Immunol $32: 257-281$

Subramanian A, Fong C-Y, Biswas A, Bongso A (2015) Comparative characterization of cells from the various compartments of the human umbilical cord shows that the Wharton's Jelly compartment provides the best source of clinically utilizable mesenchymal stem cells. PLoS One 10: e0127992

Sun J, Han Z, Liao W, Yang SG, Yang Z, Yu J, Meng L, Wu R, Han ZC (2011) Intrapulmonary delivery of human umbilical cord mesenchymal stem cells attenuates acute lung (FOXP3) + 
regulatory $\mathrm{T}$ cells and balancing anti- and pro-inflammatory factors. Cell Physiol Biochem 27:587-596

Tam K, Cheyyatraviendran S, Venugopal J, Biswas A, Choolani M, Ramakrishna S, Bongso A, Fong CY (2014) A nanoscaffold impregnated with human Wharton's Jelly stem cells or its secretions improves healing of wounds. J Cell Biochem 115:794-803

Todeschi MR, El Backly R, Capelli C, Daga A, Patrone E, Introna M, Cancedda R, El Backly R, Capelli C, Daga A, Patrone E, Introna M, Cancedda R, Mastrogiacomo M (2015) Transplanted umbilical cord mesenchymal stem cells modify the in vivo microenvironment enhancing angiogenesis and leading to bone regeneration. Stem Cells Dev 24:1570-1581

Troyer DL, Weiss ML (2008) Concise review: Wharton's jelly-derived cells are a primitive stromal cell population. Stem Cells 26:591-599

Tsai P, Fu T-W, Arthur Chen Y-M, Ko T, Chen T, Shih Y, Hung S, Fu Y (2009) The therapeutic potential of human umbilical mesenchymal stem cells from Wharton's Jelly in the treatment of rat liver fibrosis. Liver Transpl 15:484-495

Tsai P-J, Wang H-S, Shyr Y-M, Weng Z-C, Tai L-C, Shyu J-F, Chen T-H (2012) Transplantation of insulin-producing cells from umbilical cord mesenchymal stem cells for the treatment of streptozotocin-induced diabetic rats. J Biomed Sci 19:47

Ujiie Y, Gomi K, Davies JE (2015) MSC functional phenotype: Assay, age and source dependence. Sci Proc 2:1-5

Wang LL, Wang LL, Cong X, Liu G, Zhou J, Bai B, Li Y, Bai W, Li M, Ji H, Zhu D, Wu M, Liu Y (2013) Human umbilical cord mesenchymal stem cell therapy for patients with active rheumatoid arthritis: safety and efficacy. Stem Cells Dev 22:3192-3202

Wang D, Feng X, Lu LL, Konkel JE, Zhang H, Chen Z, Li X, Gao X, Lu LL, Shi S, Chen W, Sun L (2014a) A CD8 T cell/indoleamine 2,3-dioxygenase axis is required for mesenchymal stem cell suppression of human systemic lupus erythematosus. Arthritis Rheumatol 66:2234-2245

Wang G, Li Y, Wang Y, Dong Y, Wang FS, Ding Y, Kang Y, Xu X (2014b) Roles of the co-culture of human umbilical cord Wharton's jelly-derived mesenchymal stem cells with rat pancreatic cells in the treatment of rats with diabetes mellitus. Exp Ther Med 8:1389-1396

Wang D, Li J, Zhang Y, Zhang M, Chen J, Li X, Hu X, Jiang S, Shi S, Sun L, Wang D, Li J, Zhang Y, Zhang M, Chen J, Li X, Hu X, Jiang S, Shi S, Sun L (2014c) Umbilical cord mesenchymal stem cell transplantation in active and refractory systemic lupus erythematosus: a multicenter clinical study. Arthritis Res Ther 16:R79

Wang P, Liu X, Zhao L, Weir MD, Sun J, Chen W, Man Y, Xu HHK (2015) Bone tissue engineering via human induced pluripotent, umbilical cord and bone marrow mesenchymal stem cells in rat cranium. Acta Biomater 18:236-248

Wharton TW (1656) Adenographia. Translated by S. Freer 1996. Oxford University Press, Oxford, UK, p 243

Wu S, Ju GQ, Du T, Zhu YJ, Liu GH (2013) Microvesicles derived from human umbilical cord Wharton's Jelly mesenchymal stem cells attenuate bladder tumor cell growth in vitro and in vivo. PLoS One 8:1-12

Wu K-H, Wu H-P, Chao W-R, Lo W-Y, Tseng P-C, Lee C-J, Peng C-T, Lee M-S, Chao Y-H (2016) Time-series expression of toll-like receptor 4 signaling in septic mice treated with mesenchymal stem cells. Shock 45:634-640

Xie Z, Hao H, Tong C, Cheng Y, Liu J, Pang Y, Si Y, Guo Y, Zang L, Mu Y, Han W (2016) Human umbilical cord-derived mesenchymal stem cells elicit macrophages into an anti-inflammatory phenotype to alleviate insulin resistance in type 2 diabetic rats. Stem Cells 34:627-639

Xu R, Zhang Z, Wang FS (2012) Liver fibrosis: mechanisms of immune-mediated liver injury. Cell Mol Immunol 9:296-301

Yan C, Song X, Yu W, Wei F, Li H, Lv M, Zhang X, Ren X (2016) Human umbilical cord mesenchymal stem cells delivering sTRAIL home to lung cancer mediated by MCP-1/CCR2 axis and exhibit antitumor effects. Tumor Biol 37:8425-8435 
Yang X, Li Z, Ma Y, Gao J, Liu S, Gao Y, Wang G (2014) Human umbilical cord mesenchymal stem cells promote carcinoma growth and lymph node metastasis when co-injected with esophageal carcinoma cells in nude mice. Cancer Cell Int 14:93

Yannarelli G, Dayan V, Pacienza N, Lee CJ, Medin J, Keating A (2013) Human umbilical cord perivascular cells exhibit enhanced cardiomyocyte reprogramming and cardiac function after experimental acute myocardial infarction. Cell Transplant 22:1651-1666

Zebardast N, Lickorish D, Davies JE (2010) Human umbilical cord perivascular cells (HUCPVC) A mesenchymal cell source for dermal wound healing. Organogenesis 6:197-203

Zhang H-C, Liu X-B, Huang S, Bi X-Y, Wang H-X, Xie L-X, Wang Y-Q, Cao X-F, Lv J, Xiao F-J, Yang Y, Guo Z-K (2012a) Microvesicles derived from human umbilical cord mesenchymal stem cells stimulated by hypoxia promote angiogenesis both in vitro and in vivo. Stem Cells Dev 21:3289-3297

Zhang Q, O'Hearn S, Kavalukas SL, Barbul A (2012b) Role of high mobility group Box 1 (HMGB1) in wound healing. J Surg Res 176:343-347

Zhang Z, Fu J, Xu X, Wang S, Xu R, Zhao M, Nie W, Wang X, Zhang J, Li T, Su L, Wang F-S (2013a) Safety and immunological responses to human mesenchymal stem cell therapy in difficult-to-treat HIV-1-infected patients. AIDS 27:1283-1293

Zhang W, Liu X, Yang L, Zhu D, Zhang Y, Chen Y, Zhang H-Y (2013b) Wharton's jelly-derived mesenchymal stem cells promote myocardial regeneration and cardiac repair after miniswine acute myocardial infarction. Coron Artery Dis 24:549-558

Zhou C, Li M, Qin A, Lv S-X, Wen-Tang, Zhu X-Y, Li L-Y, Dong Y, Hu C-Y, Hu D-M, Wang S-F (2013a) Reduction of fibrosis in dibutyltin dichloride-induced chronic pancreatitis using rat umbilical mesenchymal stem cells from Wharton's jelly. Pancreas 42:1291-1302

Zhou Y, Xu H, Xu W, Wang B, Wu H, Tao Y, Zhang B, Wang M, Mao F, Yan Y, Gao S, Gu H, Zhu W, Qian H (2013b) Exosomes released by human umbilical cord mesenchymal stem cells protect against cisplatin-induced renal oxidative stress and apoptosis in vivo and in vitro. Stem Cell Res Ther 4:34 